\title{
BOOSTER INFLECTOR SPECIFICATIONS
}

\section{BOOSTER TECHNICAL NOTE \\ NO. 159}

\author{
C. J. GARDNER
}

FEBRUARY 28, 1990

\section{ALTERNATING GRADIENT SYNCHROTRON DEPARTMENT BROOKHAVEN NATIONAL LABORATORY \\ UPTON, NEW YORK 11973}




\title{
Booster Inflector Specifications
}

\author{
C. J. Gardner
}

February 26, 1990

\section{Location and Radius of Curvature}

\subsection{Given Information}

Based on Frank Karl's measurements and calculations [3], the distance, $d+a$, and angle, $\theta$, shown in Figure 1 are:

$$
\begin{aligned}
d+a & =66.937 \text { inches }=1.70020 \text { meters, } \ldots . \\
\theta & =14^{\circ} 59^{\prime} 57.6^{\prime \prime}=14.99933^{\circ} .
\end{aligned}
$$

\subsection{Some Algebra}

Refering to Figure 1, the distance of the trajectory from the beam center line at the exit of the inflector is $b_{1}$, and the angle of the trajectory with respect to the beam center line at this point is $\phi$. The distance from the trajectory at the inflector exit to the upstream steel face of quad QHC4 is $d$. We shall, for the moment, consider $b_{1}, \phi$, and $d$ to be variables and calculate other parameters in Figure 1 in terms of these parameters.

The point $\left(x_{0}, y_{0}\right)$ shown in Figure 1 is the center of curvature of the heavy ion trajectory in the inflector. It is defined by the intersection of lines $y=-m_{1} x-b_{1}$ and $y=m_{2} x-b_{2}$. The slopes of these lines are

$$
m_{1}=\cot \phi, \quad m_{2}=\cot \theta .
$$

The point $\left(x_{1}, y_{1}\right)=\left(0,-b_{1}\right)$ is the location of the trajectory at the exit of the inflector. 
The point $\left(x_{2}, y_{2}\right)$ is the location of the trajectory at the entrance of the inflector and is the intersection of lines $y=-(x-a) / m_{2}$ and $y=m_{2} x-b_{2}$.

Thus we have

$$
\begin{gathered}
R_{1}=\sqrt{\left(x_{1}-x_{0}\right)^{2}+\left(y_{1}-y_{0}\right)^{2}}=x_{0} \sqrt{1+m_{1}^{2}} \\
R_{2}=\sqrt{\left(x_{2}-x_{0}\right)^{2}+\left(y_{2}-y_{0}\right)^{2}}=\left(x_{2}-x_{0}\right) \sqrt{1+m_{2}^{2}}
\end{gathered}
$$

and

$$
x_{0}=\left(\frac{b_{2}-b_{1}}{m_{1}+m_{2}}\right), \quad x_{2}=\left(\frac{m_{2} b_{2}+a}{1+m_{2}^{2}}\right) .
$$

Now since we require the trajectory to be a circular arc we must have

$$
R_{1}=R_{2}=R .
$$

Using (3-6) in (7) we then find

$$
\begin{gathered}
b_{2}=\frac{a \sin \theta \sin (\phi+\theta)+b_{1}(\sin \phi+\sin \theta)}{\sin \theta-\sin \theta \cos (\phi+\theta)}, \\
x_{0}=\frac{\sin \phi \sin \theta}{\sin (\phi+\theta)}\left(b_{2}-b_{1}\right), \\
x_{2}=b_{2} \cos \theta \sin \theta+a \sin ^{2} \theta \\
R=\frac{\sin \theta}{\sin (\phi+\theta)}\left(b_{2}-b_{1}\right) .
\end{gathered}
$$

\subsection{Position and Angle of E.O. at Inffector Exit}

On page 4 of Reference 1 it is shown that the position and angle of the bumped equilibrium orbit at a distance $d_{1}=30 \mathrm{~cm}$ upstream of the upstream steel face of quad QHC4 are given by

$$
X_{0}=-7.738 \theta_{k}, \quad X_{0}^{\prime}=-1.232 \theta_{k}
$$

where $X_{0}$ is given in meters and $\theta_{k}$ is given in radians. Given these relations we must find $\theta_{k}$ such that the bumped orbit has position $X=-b_{1}$ at the inflector exit. Since the space between QVC3 and QHC4 is a drift, we have

$$
X_{0}=X+X_{0}^{\prime}\left(d-d_{1}\right)=-b_{1}+X_{0}^{\prime}\left(d-d_{1}\right) .
$$


Inserting equations (12) and solving for $\theta_{k}$ we find

$$
\theta_{k}=\frac{b_{1}}{7.738-1.232\left(d-d_{1}\right)} .
$$

In order for the heavy ion trajectory at the exit of the inflector to be tangent to the bumped orbit we must also have

$$
\sin \phi=-X_{0}^{\prime}
$$

\subsection{Some Numbers}

To insure that the radius of curvature, $R$, is not too small (which is necessary to keep the inflector voltage down), and to insure that beam injection occurs as close as possible to the horizontal beta maximum, the distance, $d$, from the exit of the inflector to the upstream steel face of quad QHC4 must be as small as possible. This requires eliminating the dipole corrector package upstream of quad QHC4 and shortening the bellows there. If this is done, then $\mathrm{V}$. Badea finds that $d$ should be no shorter than $35.000-8.375=26.625$ inches. We therefore take $d=26.625$ inches $(0.67628$ meters $)$ and hence $a=(d+a)-d=40.312$ inches $(1.02392$ meters).

We also require that the inflector septum be outside the booster's limiting aperture, which is 2.0 inches $(50.8 \mathrm{~mm})$ at the horizontal beta maximum of 13.9 meters. Since the horizontal beta at the inflector exit is 10.9 meters, the septum must be at least $(10.9 / 13.9)^{1 / 2}(2.0)=1.77$ inches $(45.0 \mathrm{~mm})$ from the beam centerline. Allowing a margin of $2.5 \mathrm{~mm}$ we therefore take the septum to be $47.5 \mathrm{~mm}$ from the beam centerline.

The nominal trajectory of the heavy ions in the inflector will be $8.5 \mathrm{~mm}$ from the septum, which coincides with the center of the $17.0 \mathrm{~mm}$ gap between the septum and cathode. Allowing $1.0 \mathrm{~mm}$ for the septum thickness, we therefore take $b_{1}=47.5+1.0+8.5=57.0 \mathrm{~mm}$. (Y. Y. Lee points out that in order to fill as much of the available phase space as possible, the incoming beam should be as close to the septum as possible without scraping it. Modeling by S. Y. Lee then indicates that the incoming beam should be $5.0 \mathrm{~mm}$ from the septum, which is $3.5 \mathrm{~mm}$ to the inside of the nominal trajectory. We plan to use correction dipoles upstream of the inflector to move the beam $3.5 \mathrm{~mm}$ to the inside of the nominal trajectory.) 
Using these results in equation (14) we find $\theta_{k}=7.836 \times 10^{-3}$, and hence $X_{0}^{\prime}=9.654 \times 10^{-3}, \phi=9.654 \mathrm{mrad}$. This means that at the upstream face of quad QHC4 the bumped orbit is $63.5 \mathrm{~mm}$ ( 2.50 inches) from the beam centerline.

Now using

$$
b_{1}=57.0 \mathrm{~mm}, \quad \phi=9.654 \mathrm{mrad}, \quad a=1.02392 \text { meters }
$$

in equations (8-11) we find

$$
\begin{gathered}
R=8.74123 \text { meters }, \quad L=R(\phi+\theta)=2.37274 \text { meters } \\
x_{0}=0.08439 \text { meters }, \quad y_{0}=-8.79783 \text { meters } \\
x_{2}=2.34669 \text { meters }, \quad y_{2}=-0.35442 \text { meters }
\end{gathered}
$$

\section{Voltage Requirement}

Let $V$ and $D=1.7 \mathrm{~cm}$ be the potential difference and distance between the septum and the cathode of the inflector. Then in order for an ion with charge $e Q$ and mass $m$ to move with speed $c \beta$ along a circular arc of radius $R$, we must have

$$
\frac{m c^{2} \gamma \beta^{2}}{R}=\frac{e Q V}{D}
$$

Thus the required inflector voltage is given by

$$
e V=\frac{D}{Q R} m c^{2} \gamma \beta^{2} \approx \frac{D}{Q R} m c^{2} \beta^{2}
$$

Now, as discussed in Reference 2, we have

$$
\frac{1}{2} m c^{2} \beta^{2} \approx\left(1+Q_{t}\right) e V_{t}
$$

where $V_{t}$ is the Tandem (MP7) voltage and $Q_{t}$ is the most probable charge state for ions after stripping inside the Tandem.

Putting (22) in (21) we then find

$$
V \approx \frac{2 D\left(1+Q_{t}\right)}{Q R} V_{t}
$$


Thus, using $D=1.7 \mathrm{~cm}, R=8.74123 \mathrm{~m}$, and $V_{t}=15 \mathrm{MV}$ in (23) we find

$$
V \approx 58.3\left(1+Q_{t}\right) / Q \mathrm{kV}
$$

A. Soukas believes that this voltage requirement does not pose any serious power supply or cable problems.

(Note that the gap, $D$, between septum and cathode of the inflector has been reduced from the original design value of $2.0 \mathrm{~cm}$ to $1.7 \mathrm{~cm}$. This was suggested by $\mathrm{Y}$. Y. Lee as way to lower the voltage requirement. He does not believe this reduction in the horizontal aperture of the inflector poses any aperture problems. Experience has shown that a $2.0 \mathrm{~cm}$ gap is adequate for the inflector currently used to inject heavy ions into the $\Lambda$ GS. Here one matches the incoming beam to the AGS acceptance which has a horizontal beta of 19.0 meters at the inflector exit. In the booster the horizontal beta at the inflector exit is 10.9 meters so that in princple one only needs a $(10.9 / 19.0)^{1 / 2}(2.0)=1.5 \mathrm{~cm}$ gap to match the incoming beam to the Booster acceptance.)

\section{References}

1. S. Y. Lee, Booster Injection Scenarios and Orbit Bump Requirements, AGS Booster Technical Note No. 155, December 18, 1989.

2. R. Thomas, Voltage Required on the Booster Electrostatic Septum, Memo to A. Soukas, January 30, 1990.

3. F. Karl, Memo, January 30, 1990. 
Booster Electrostatic Inflection

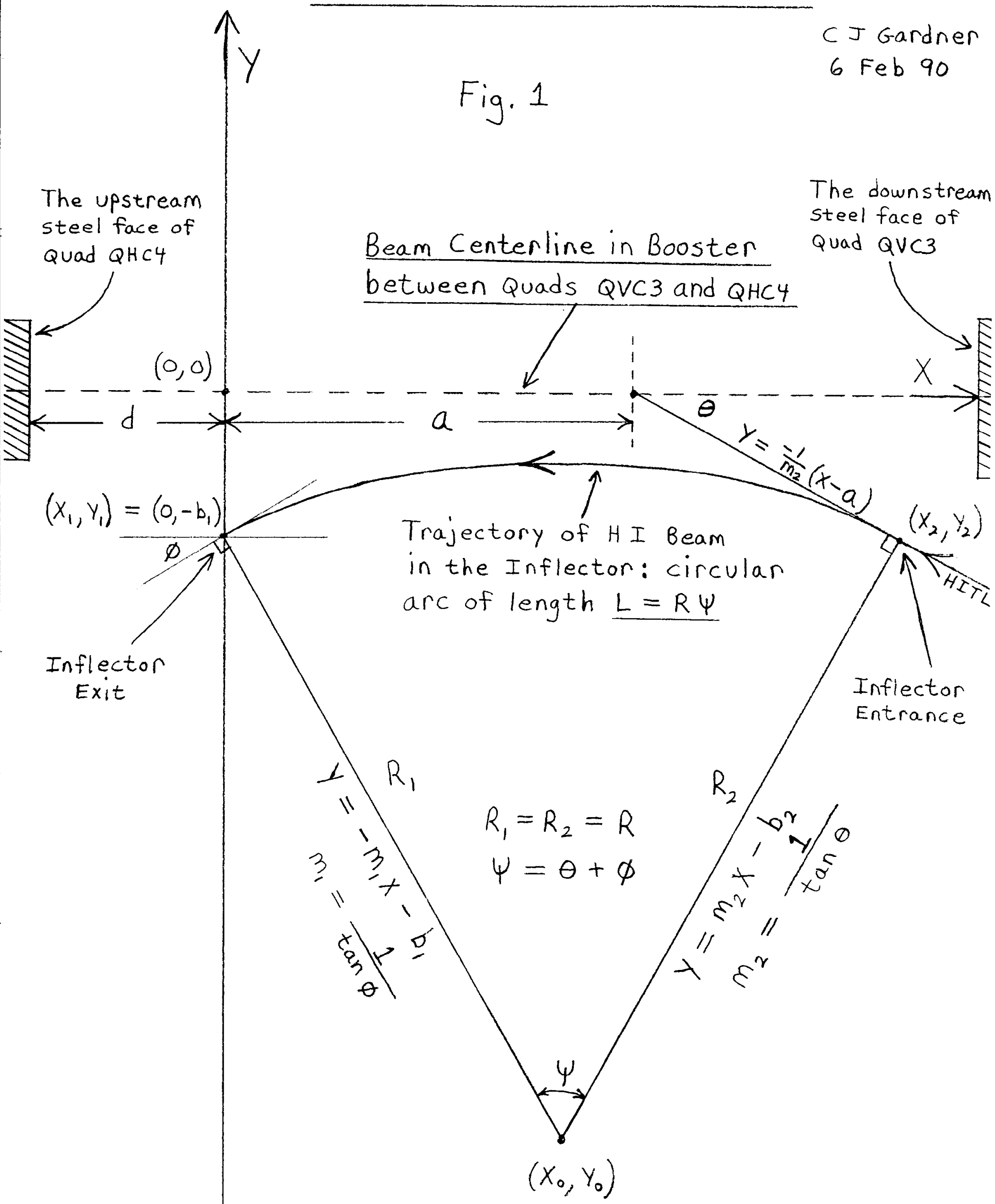


Inflictor Detail

cU Gardner

26 Feb 90

$-1-\cdots-$ Beam Centerline $-\ldots-\cdots$

$57.0 \mathrm{~mm}$

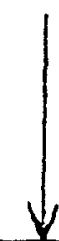

$\sim 1 \mathrm{~mm}$

$1.7 \mathrm{~cm} \mathrm{gap}$
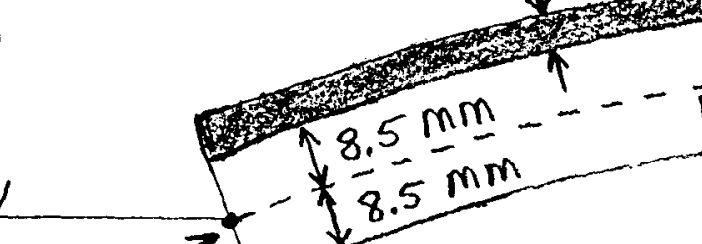

Nominal Trajectory

This is the

point $\left(x_{1}, y_{1}\right)$

shown in

figure 1. 


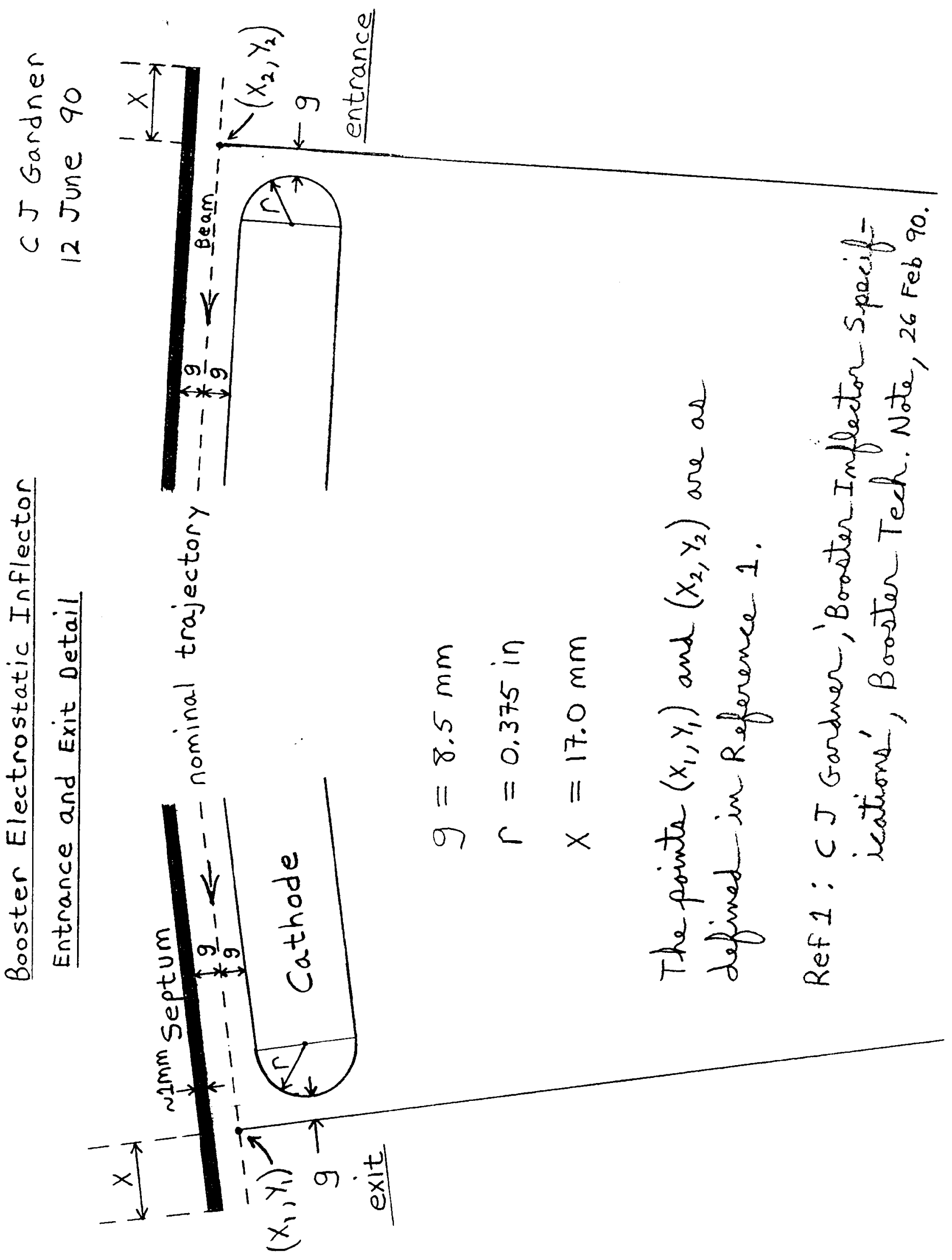

